\title{
PROTOCOLO PARA GERENCIAMENTO HIERÁRQUICO DE REDES DE COMPUTADORES E DE TELECOMUNICAÇÕES
}

\author{
Marcial Porto Fernandez, Gardel Moreira Delfino e Aloysio de Castro P. Pedroza
}

\begin{abstract}
Resumo - Os sistemas de gerenciamento de redes de computadores freqüentemente são estruturados segundo uma arquitetura simples, constituída por processos de gerência (gerente-agente). A gerência de redes de telecomunicações, pela sua dimensão e complexidade, exige um sistema de gerenciamento com maior capacidade, impossível de se obter com apenas um gerente. O TMN (Telecommunications Management Network), que é um modelo para gerência de telecomunicações, prevê o uso de vários gerentes organizados segundo uma arquitetura hierárquica. Este trabalho apresenta um estudo dos problemas de gerenciamento de grandes redes de telecomumicações e propõe um protocolo para um Sistema de Gerenciamento Hierárquico (SGH). Apesar de utilizarmos como exemplo o gerenciamento de telecomumicações. o sistema SGH pode ser adaptado para o ambiente Internet. O protocolo SGH é especificado através da linguagem de descrição formal Estelle e suas funcionalidades são verificadas utilizando-se um modelo de teste.
\end{abstract}

Palavras chaves - gerenciamento de redes, gerenciamento de telecomunicações, protocolo

\begin{abstract}
Abstact - A computer network management system usually presents a management process (manager-agent). The management of large networks demands more computer resources and management system capacity, impossible to achieve with just one manager. The TMN (Telecommumications Management Network) standard, that intends to standardize management systems for telecommunication networks offering those resources, foresees the use of several managers orgamized in a hierarchical architecture. This work presents a study of the problems in managing large networks and proposes a protocol for a Hierarchical Management System (SGH). Although we use an example of telecommunication network, the SGH system is suitable for Internet. The SGH protocol is specified using the formal description language Estelle and its functionality is verified using a test model.
\end{abstract}

Keywords - network management, telecommunication, protocol

Os autores são do Grupo de Teleinformática e Automação (GTA) COPPE/PEE - Programa de Engenharia Elétrica da Universidade Federal do Rio de Janeiro (UFRJ). Aloysio Pedroza também é do Depto. de Eletrônica/EE-UFRJ. E-mails: marcial@gta.ufrj.br, gardel@gta.ufrj.br e aloysio@gta.ufrj.br.

Editores responsáveis: Max Henrique M. Costa (Ad Hoc) e José Augusto M. Suruagy (Editor de Área). Submetido em 10/1998: revisado em 11/1999 e 12/2000; aceito em 11/2001.

92

\section{INTRODUÇÃO}

Os sistemas de gerenciamento de redes de comunicação utilizam uma mesma filosofia: um elemento principal, denominado gerente, deve reunir informações e realizar comandos de controle do sistema, enquanto os demais elementos, os agentes, adquirem estas informações e executam as ordens dadas pelo gerente.

Esta arquitetura simples apresenta deficiências quando há um aumento da quantidade de elementos a serem gerenciados. A adição de elementos agentes aumenta a necessidade de processamento no gerente, bem como o tráfego de dados nos seus canais de comunicação. Este é o caso do gerenciamento de redes de telecomunicações, nas quais a quantidade de elementos gerenciados pode chegar a vários milhões.

A norma TMN[1], publicada em 1992, especifica uma arquitetura para sistema de gerência integrada de redes de telecomunicações, contrapondo-se aos sistemas proprietários, existentes até então. O ITU-T (International Telecommunication Union - Telecommunication Standardization Sector) reconheceu a necessidade da concepção de um sistema com vários gerentes organizados de forma hierárquica, mas não definiu como esse tipo de gerência seria implementado. Por isso, a maioria das implementações existentes acabaram utilizando soluções proprietárias.

O presente trabalho define um protocolo que implementa um sistema de gerenciamento hierárquico. A arquitetura proposta, adequada ao controle de grandes redes de telecomunicações, restringe o gerenciamento a pequenos grupos, mas permite a sincromzação das ações de gerenciamento em todo o sistema. Utilizamos a arquitetura TMN para processar todas as informações de gerenciamento e realizar o controle nos elementos gerenciados. Para verificar as funcionalidades desta proposta, foi realizado um protótipo utilizando-se a linguagem de descrição formal Estelle.

A seção 2 traz uma visão dos problemas para gerenciamento de grandes redes de comunicação e destaca algumas propostas encontradas na literatura. Na seção 3, encontram-se a especificação do Sistema de Gerenciamento Hierárquico (SGH) proposto, as primitivas do serviço oferecido e o protocolo correspondente. A seção 4 apresenta o projeto do sistema $\mathrm{SGH}$, o método adotado, o modelo correspondente, a especificação formal, a simulação, o teste e a implementação obtida. Finalmente, a seção 5 apresenta as conclusões do trabalho e sugere temas para trabalhos futuros.

\section{ARQUITETURA DE GERENCIAMENTO}

As arquiteturas de gerenciamento podem ser divididas em três grupos: tradicional, um gerente e vários agentes; 
distribuída, vários gerentes distribuindo os agentes: e hierárquica, vários gerentes e agentes relacionados hierarquicamente. Esta seção apresenta os problemas para gerenciar uma grande rede de telecomunicações e mostra algumas técnicas de gerenciamento hierárquico e conceitos de gerenciamento distribuídos utilizados em nosso trabalho.

\subsection{PROBLEMAS DE GERENCIAMENTO DE UMA REDE DE GRANDES DIMENSÕES}

O principal problema de uma grande rede é não poder ser gerenciada apenas por um gerente. Mesmo que seja admitido o aumento da capacidade dos novos equipamentos, sempre teremos um limite, ora por causa do processamento, ora por causa da velocidade do canal de comunicação. Assim o sistema deve ser flexível, escalonável, podendo a sua capacidade ser aumentada de acordo com a demanda por recursos de gerenciamento.

Um sistema distribuído, com vários gerentes, cada um deles gerenciando um grupo de agentes, poderia solucionar - problema da capacidade, mas daí surge um novo problema: a existéncia de vários gerentes pode provocar um conflito de autoridade. Portanto, cada gerente deve saber o que pode controlar e a quem deve se reportar, de forma que o sistema fique coerente e não haja conflito de autoridade entre gerentes diferentes. Em um sistema de grande porte, não se pode vincular rigidamente um agente a um gerente, pois no caso de um gerente ficar inoperante, deve-se ter a possibilidade de outro gerente assumir o controle. O sistema deve ser flexível também quanto à possibilidade de acrescentar elementos gerenciados dinamicamente, à medida em que forem ativados, assim como retirá-los caso estes sejam desativados.

Um sistema hierárquico deve também respeitar a responsabilidade e autoridade das empresas que operam os sistemas de telecomunicação. Como é comum haver diversas empresas operando as telecomunicações em uma dada região, algumas atendendo ligações locais, dentro desta mesma região, e outras para ligações de longa distância no país e ligações internacionais, é necessário respeitar a autoridade sobre a área de atuação. Por exemplo, uma empresa que atua em longa distância não pode controlar um canal dentro de uma dada região, quando este for de responsabilidade de uma empresa local. A empresa de longa distância, no entanto, poderá definir parâmetros para os canais de comunicação desta empresa local de forma a garantir a qualidade da comunicação global.

\subsection{TÉCNICAS PARA GERENCIAMIENTO DE SISTEMAS DE GRANDE PORTE}

A seguir, apresentamos resumidamente várias técnicas encontradas na literatura que visam à implementação de um grande sistema de gerenciamento. No primeiro item, apresentamos técnicas de gerenciamento hierárquico no ambiente Internet e, no segundo item, apresentamos os vários conceitos de gerenciamento distribuído que forneceram subsídios teóricos formais ao nosso trabalho.

\subsubsection{TÉCNICAS DE SISTEMAS DE GERENCIAMENTO HIERÁRQUICO}

Analisamos aqui algumas técnicas de gerenciamento hierárquico no ambiente Internet. Apresentamos uma evolução histórica de três implementações de gerenciamento hierárquico usando o protocolo SNMP[2].

\subsubsection{MANAGER-TO-MANAGER MIB}

A primeira técnica de sistema com mais de um gerente implementada no padrão Internet e foi chamada Managerto-Manager MIB, especificada por CASE et al. [3]. Um gerente secundário concentra informações oriundas dos agentes e replica os dados de gerenciamento de sua MIB, como alarme, eventos e notificação, no gerente principal. Além disso, este elemento principal realiza uma sincronização da base de dados evitando, por exemplo, que dois gerentes escrevam na MIB do mesmo agente informaçôes diferentes. A Manager-to-Manager MIB está implementada apenas na versão 2 do SNMP[4].

O objetivo principal dessa técnica é o de permitir que gerentes secundários instalados remotamente, e com linhas de comunicação lenta, sejam gerenciados, sem degradar a eficiência do canal de dados. O sistema Manager-toManager não é apropriado para gerenciar grandes redes, pois não reduz o processamento de informações no gerente principal, apenas diminui a frequiência de envio e a quantidade de dados transmitidos para o gerente principal. A função de monitoração do gerente secundário apenas testa valores dentro de certos limites inferiores e superiores e nenhum cálculo mais complexo necessário para tomar decisão é realizado [5]. Finalmente, esta técnica não tem capacidade para realizar controle hierárquico completo, pois implementa, no máximo, mais um nível intermediário entre o gerente e um conjunto de agentes.

\subsubsection{GERENCIAMENTO HIERÁRQUICO - SUB MANAGER}

O Sub Manager é uma técnica proposta por SIEGL e TRAUSMUTH [6] para o ambiente Internet. Ela amplia o conceito da Manager-to-Manager MIB, aumentando a funcionalidade e permitindo um número maior de núveis hierárquicos.

A idéia básica é criar um dispositivo entre o gerente e agente chamado Sub-Manager. Esse dispositivo é constituído por um controle e três tabelas: uma chamada SubMgrEntry, que armazena os dados de entrada vindos dos agentes; uma outra chamada SubMgrOps, com a lista de procedimentos (como um script de funções de gerenciamento); e outra chamada SubMgrValue, com os resultados do cálculo de SubMgrEntry pelos procedimentos de SubMgrops.

Esta técnica também não foi utilizada em nosso sistema, pois, assim como a Manager-to-Manager MIB, não diminui o processamento no gerente principal. 


\subsubsection{GERENCIAMENTO HIERÁRQUICO - META MIB}

A Meta-MIB é uma técnica para representar a configuração física no ambiente Internet, considerando conceitos de gerenciamento hierárquico e descentralizado. É utilizada no Projeto WILMA e descrita por SCHALLER [7]. A idéia é definir três novas MIBs:

Tabela de Componentes: especificando o tipo de equipamento em cada endereço IP, como computador, roteador, modem, etc.

Tabela de Conexões: especificando todas as conexões de equipamentos, por exemplo, roteador 1 com modem 3 , estação4 com Hub2.

Tabela de Agentes: especificando o estado em nível maior de abstração, tomando como base informações dos agentes. Por exemplo, o estado de uma linha de comunicação é a combinação do estado de dois roteadores e dois modenis.

Esta técnica permite a definição de um elemento abstrato que possibilita criar vários níveis hierárquicos. Aproveitamos o conceito de Meta-MIB, em nosso sistema, conservando inclusive a denominação.

\subsubsection{CONCEITOS DE SISTEMAS DE GERENCIAMENTO DISTRIBUÍDO}

A implementação de Meta-MIB propicia a criação de uma arquitetura hierárquica, mas ainda rígida. Para conferir flexibilidade à arquitetura de nosso sistema, utilizamos conceitos de Sistemas Distribuídos, os quais descrevemos a seguir.

\subsubsection{GERÊNCIA DE DOMÍNIOS}

A Gerência de Domínios é proposta por SLOMAN et al [8] e define os relacionamentos entre os diversos elementos de uma rede. Em um grande sistema de gerenciamento, é difícil controlar e visualizar todos os elementos conectados à rede. Qualquer gerente, sistema ou operador humano, deve agrupar vários objetos gerenciados em um único objeto. Com base nisso, os autores definem os conceitos de objeto gerenciado, objeto composto e objeto gerenciador, apresentados a seguir.

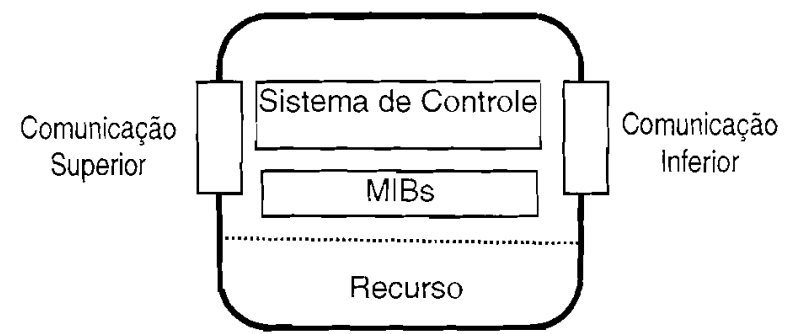

Figura 1. Modelo de um objeto gerenciado

O objeto gerenciado é definido como uma entidade que dispõe de recursos a serem gerenciados. De forma geral, podemos representar o objeto gerenciado como mostrado na figura 1. O elemento principal é o núcleo de gerenciamento, que é composto pelo software de controle e as tabelas de dados (MIBs). Este núcleo interage com o meio exterior através de interfaces de comunicação com o dispositivo superior, com o dispositivo inferior e com o recurso local.

O objeto composto é definido como um conjunto de objetos gerenciados que possui um ou mais objetos gerenciadores (gerentes). Este conjunto é considerado como um único objeto gerenciado que encapsula todos os objetos internos. Um exemplo de objeto gerenciado composto é mostrado na figura 2. A conexão de objetos gerenciados compostos forma uma árvore hierárquica com relações de responsabilidade e autoridade rígidas entre os objetos.

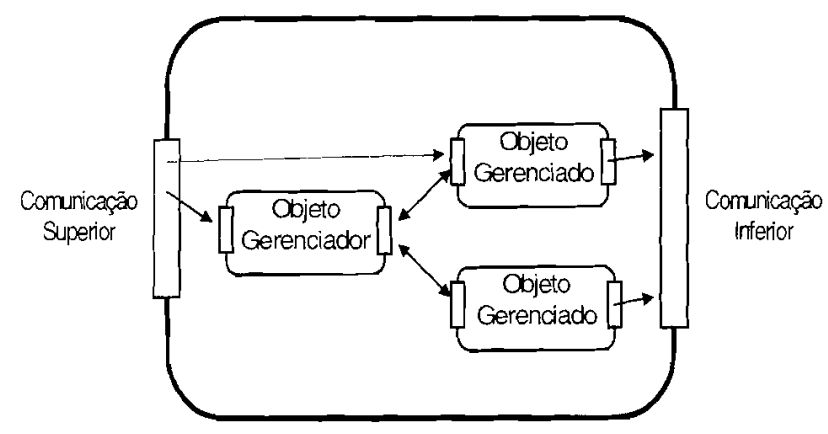

Figura 2. Modelo de um domínio

O objeto gerenciador consolida todos as informações colhidas pelos objetos gerenciados e representa todo esse conjunto de informações como um único objeto. Deste modo, a situação de todos os sub-objetos coletados pelo objeto gerenciado é um indicativo global do domínio (representado na figura com linha cheia). Se um dispositivo superior necessitar de uma informação específica de um objeto gerenciado, pode solicitar diretamente ao objeto que guarda as informações (representado na figura com linha tracejada).

\subsubsection{DIRETRIZ DE GERENCIAMENTO}

A Diretriz de Gerenciamento também é uma proposta de SLOMAN et al [8]. O gerenciamento de sistemas distribuídos envolve as funções de monitorar as atividades, tomar decisões sobre gerenciamento e executar ações de controle para modificar o comportamento do sistema considerado. Pela própria filosofia desse tipo de sistema, o gerente deve ter autonomia para decidir as ações que devem ser tomadas, sem precisar receber ordem explícita de um gerente de nível superior. Estas decisões, no entanto, dentro do sistema distribuído, com vários objetos executando a função de gerência, precisam de diretrizes que orientem sua realização.

Um sistema de gerenciamento com grande quantidade de objetos não pode ter uma diretriz para cada objeto individualmente. Assim, é necessário definir vários domínios, agrupando diversos objetos com funções semelhantes, para os quais diretrizes devem ser especificadas.

O domínio, portanto, pode ser definido como um objeto ou um conjunto de objetos gerenciadores e gerenciados, ao qual se aplica uma diretriz.

A especificação de diretrizes por um gerente humano inicia-se no nível mais elevado de abstração e vai sendo refinada a cada nível que desce. Observe que uma diretriz não especifica necessariamente o que fazer em dada situação, mas ela determina os parâmetros de decisão. A 
diretriz não é simplesmente um valor que deve ser atendido. Em um sistema heterogêneo, constituído por diversos equipamentos de diversos fabricantes, essas diretrizes poderão tomar valores distintos em função das características particulares de cada objeto gerenciado.

Outra proposta de gerenciamento de diretrizes foi feita por WIES [9]. A idéia básica é semelhante, apenas complementa a idéia anterior, classificando as diretrizes segundo critérios mais específicos.

\subsubsection{DELEGAÇÃO DE GERENCIAMENTO}

A delegação de gerenciamento, que é proposta por GOLDSZMIDT e YEMINI [10], especifica um modelo de sistema de gerenciamento flexível, isto é, um sistema adequado para controlar uma rede que se modifica ao longo do tempo.

Na proposta dos autores, parte do código executável de um gerente pode ser executada em outro dispositivo, quando o sistema detecta que está sobrecarregado. Outro tipo de delegação acontece quando um gerente sofre paralisação. Neste caso, um gerente de nível hierárquico superior assume o controle dos objetos e tem todo o poder sobre eles, inclusive de delegá-los a outro gerente.

Idéia mais complexa foi proposta por MOFFETT e SLOMAN [11] e utilizada no Projeto DOMINO: além da autoridade de poder, especifica também a autoridade de delegação. $\mathrm{O}$ sistema pode impor restrições semelhantes às organizações humanas, onde um chefe pode delegar a chefia a um subordinado, mas este não pode delegá-la a outra pessoa sem o consentimento do chefe, por exemplo.

\subsection{PROPOSTA PARA UM SISTEMA DE GERENCIAMENTO DE GRANDE PORTE}

A maioria das técnicas apresentadas para gerenciamento hierárquico no ambiente Internet são inadequadas para um sistema de gerenciamento de grande porte.

O sistema Manager-to-Manager não é apropriado para gerenciar grandes redes pois, como mencionamos anteriormente, não reduz o processamento de informações no gerente principal, apenas diminui a quantidade de dados transmitidos entre os gerentes. Além disso, não tem capacidade para realizar controle hierárquico completo, pois implementa, no máximo, dois níveis hierárquicos. $\mathrm{O}$ uso do sistema SubManager não foi utilizado pela mesma razão do sistema Manager-to-Manager, no qual inclusive se baseia. Apesar de admitir vários níveis intermediários entre gerente e agente continua a concentrar o processamento no gerente principal.

Dessas técnicas, aproveitamos a Meta-MIB, que propicia a criação de uma verdadeira arquitetura hierárquica, mesmo que ainda rígida. A tabela da Meta-MIB mais interessante para o nosso sistema é a Tabela de Agentes, que permite a representação de vários níveis hierárquicos dentro de um sistema de gerenciamento. Em nossa proposta, utilizamos uma estrutura chamada Meta-MIB, que implementa a função da Tabela de Agentes.

Nosso sistema é a combinação dessa técnica com os conceitos de Sistemas Distribuídos, que lhe proporcionam flexibilidade. A Gerência de Domínio possibilita o controle da posição dos gerentes e agentes na hierarquia. A Diretriz de Gerenciamento permite organizar os graus de liberdade de comando no sistema, definindo limites de controle para que todos os gerentes possam ter autonomia sem interferir no desempenho global do sistema. E a Delegação de Gerenciamento oferece recursos para o próprio sistema efetuar o equilíbrio de carga, alocando agentes para outros gerentes.

\section{ESPECIFICAÇÃO DE UM SISTEMA DE GERENCIAMENTO HIERÁRQUICO (SGH)}

O Sistema de Gerenciamento Hierárquico (SGH) é um módulo que funciona paralelamente ao módulo TMN e executa todas as funções descritas a seguir. Mostraremos que este sistema de gerenciamento, utilizando arquitetura hierárquica, pode controlar e monitorar uma grande quantidade de dispositivos. A base desta arquitetura está na concepção de dispositivos que admitem dois comportamentos distintos: um como gerente (em relaçāo aos diversos agentes sob seu controle) e outro como agente (em relação ao gerente que o controla).

\subsection{ESTRUTURA DO SISTEMA DE GERENCIAMENTO HIERÁRQUICO (SGH)}

O SGH está situado na camada Aplicação. O modelo de organização do sistema proposto é apresentado na figura 3 , que mostra esquematicamente a disposição dos diversos módulos. As camadas inferiores estão representadas genericamente como Meio de Comunicação. Acima delas, está a camada que oferece o serviço CMIS (Common Management Information Service) e que realiza a comunicação através do protocolo CMIP [12].

O módulo TMN executa as funções de coleta de informações dos elementos gerenciados e as consolida em suas bases de dados de gerenciamento (MIBs). Este módulo implementa todas as funções de gerenciamento de uma rede de telecomunicações. A camada de nível mais elevado foi chamada de Plataforma de Gerenciamento e consiste em um sistema que oferece as informações de gerenciamento para o operador da rede.

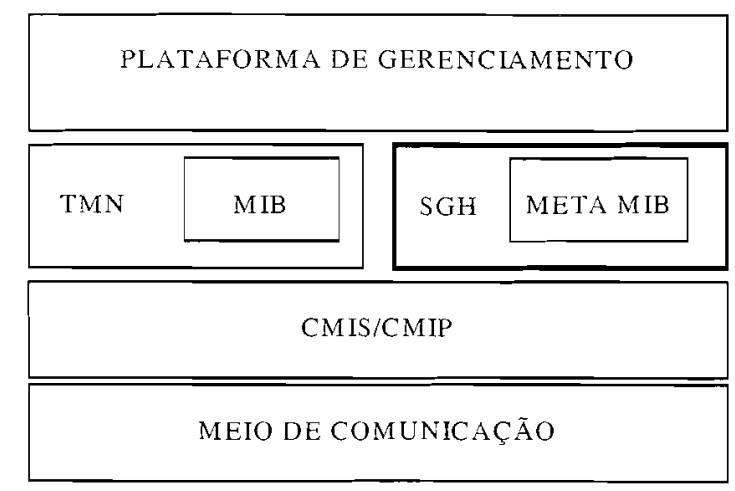

Figura 3. Arquitetura do Sistema de Gerenciamento

O módulo SGH executa as funções de Gerência de Domínio, Gerência de Diretrizes e Gerência de Delegação, conceitos detalhados na seção 2.2.2. Ele assume o comportamento de agente, fornecendo informaçôes 
(reports) e recebendo informações de gerenciamento do gerente um nível acima, utilizando os dados consolidados na Meta-MIB. A relação com a camada inferior é realizada através das primitivas do serviço CMIS. Para a camada superior, são oferecidas as primitivas do serviço SGH. A Meta-MIB apresenta para o gerente acima a imagem da rede gerenciada, segundo a visão do nível superior.

\subsection{ESTRUTURA DE DADOS DO SGH}

A estrutura de dados é mostrada na figura 4 e é composta por uma base de dados com todos os parâmetros necessários, como tabela de gerentes, tabela de agentes, tabela de diretrizes e a Meta-MIB, que serão descritas a seguir. A estrutura de controle realiza a manipulação de dados nas tabelas, oferece o serviço SGH especificado na seção 3.3 e implementa o protocolo $\mathrm{SGH}$, utilizando o serviço CMIS, que é descrito na seção 3.4 .

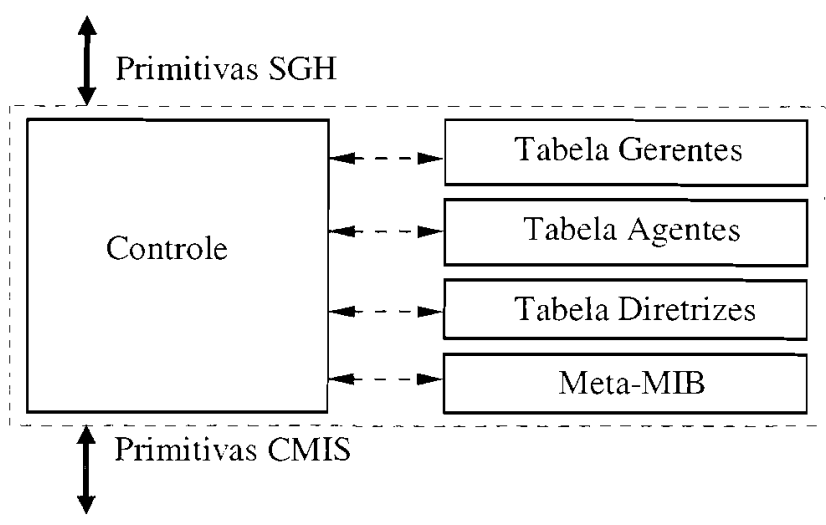

Figura 4. Estrutura de dados do $\mathrm{SGH}$

A Tabela de Gerentes (TableM) é uma lista com o endereço dos dispositivos gerentes de nível hierárquico superior, em ordem prioritária de conexão. Se o primeiro gerente não responder (estiver fora de operação, por exemplo) deve-se tentar contato com o segundo gerente da tabela e assim por diante.

A Tabela de Agentes (TableA) determina todos os objetos gerenciados por determinado gerente. Com essa lista, poderão ser realizadas as funções de delegação.

A Tabela de Diretrizes (TablePolicy) armazena os parâmetros do sistema. Usamos como referência atributos propostos na norma TMN [13]. Maiores detalhes de nossa proposta de diretrizes são apresentadas em [14].

A Meta-MIB deverá armazenar as informações de um dispositivo hipotético para o nível superior: A definição e tratamento desta MIB não é escopo do presente trabalho.

\subsection{ESPECIFICAÇÃO DO SERVIÇO OFERECIDO PELAS PRIMITIVAS SGH}

O serviço oferecido pelo conjunto de primitivas SGH é a interface com o usuário do sistema é detalhado em [14]. Estas primitivas foram selecionadas a partir de sugestões encontradas na literatura referenciada.

O serviço de Gerência de Domínio baseia-se nas especificações descritas na seção 2.2.2.1 e é constituído pelas primitivas: MInitPrin, ManagerInit, ManagerRead, AgentRead, AgentCreate e AgentElimin. As primitivas de gerência de domínio foram definidas através das sugestões indicadas por SLOMAN et al [8] e permitem a manipulação de gerentes e agentes pertencentes ao conjunto de objetos gerenciados de um determinado gerente, como, por exemplo, a inclusão de um objeto novo ou a retirada de um objeto não mais utilizado.

$O$ serviço de Gerência de Diretrizes baseia-se nas especificações descritas na seção 2.2.2.2 e é constituído pelas primitivas: PolicyMRead, PolicyARead, PolicyAChange e PolicyMLoad. As primitivas de gerência de diretrizes foram definidas também com as sugestões indicadas por SLOMAN et al [8]. Essas primitivas permitem o tratamento e manipulação de um determinado gerente. Por exemplo, permitem ler as diretrizes do gerente ou escrever diretrizes em um agente.

O serviço de Gerência de Diretrizes baseia-se nas especificações descritas na seção 2.2.2.3 e é constituído pela primitiva DelegAct. A primitiva de delegação foi definida com base nos conceitos apresentados por MOFFETT e SLOMAN [11]. Essa primitiva executa a função de delegação de agentes de um gerente para outro.

\subsection{ESPECIFICAÇÃO DO PROTOCOLO SGH}

O protocolo SGH utiliza primitivas CMIS. Como as comunicações realizadas pelo sistema são esporádicas apenas quando é necessária alguma alteração na hierarquia ou de seus parâmetros -, toda primitiva SGH começa com a primitiva de associação A-Associate e termina com a primitiva de encerramento A-Release, pertencente ao ACSE (Association Control Service Element). A especificação detalhada do protocolo de cada primitiva está mostrada em [14].

\section{PROJETO DO SISTEMA DE GERENCIAMENTO HIERÁRQUICO (SGH)}

O projeto do Sistema de Gerenciamento Hierárquico, que consiste na descrição do método e o desenvolvimento do sistema objeto deste trabalho, é apresentado a seguir. Inicialmente, descrevemos o protótipo de simulação e teste, que visa demonstrar todas as funcionalidades do sistema proposto. Em seguida, apresentamos o método adotado no projeto: a modelagem do protocolo, utilizando a técnica de Rede Predicado-Ação, e a especificação do protocolo, utilizando a linguagem de descrição formal Estelle. Finalmente, apresentamos os testes realizados na ferramenta de simulação e a implementação do protocolo em código executável.

\subsection{DESCRIÇÃO DO PROTÓTIPO}

O sistema de gerenciamento hierárquico proposto neste trabalho foi testado com a simulação do caso apresentado na figura 5. Utilizando este modelo, podem ser realizados os testes abaixo:

- Ativação de um gerente (de qualquer camada), assim como a atualização das suas tabelas.

- Inclusão e exclusão de agentes na estrutura. 

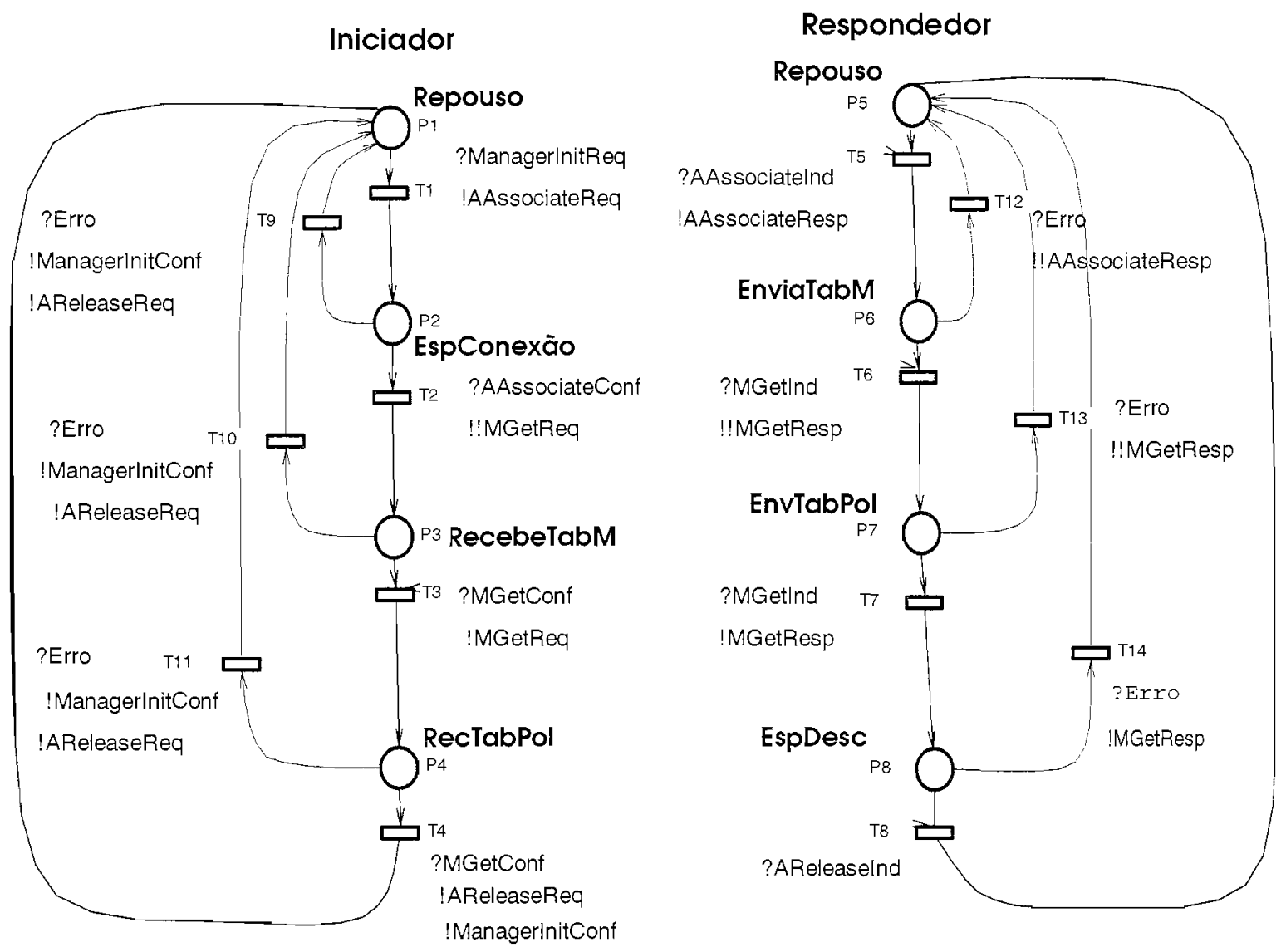

Figura 5. Rede Predicado-Ação da primitiva ManagerInit

- Mudança na Tabela de Diretrizes.

- Delegação de gerenciamento, isto é, um gerente passa para outro o controle de alguns objetos gerenciados.

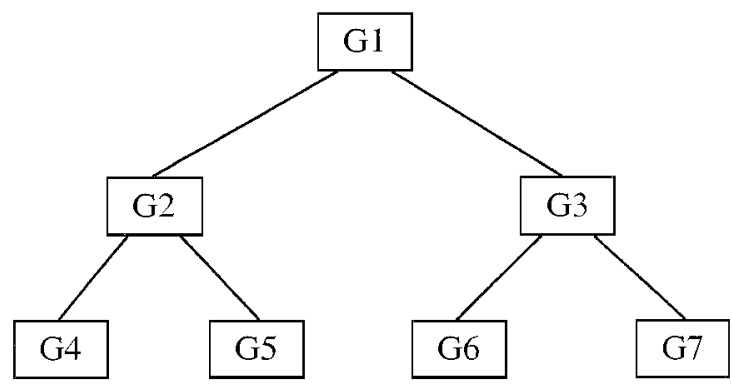

Figura 6. Proposta de Estudo de Caso

\subsection{MÉTODO DE PROJETO DO SGH}

O projeto do Sistema de Gerenciamento Hierárquico (SGH) utilizou o método de especificação em linguagem de descrição formal. Utilizamos a linguagem Estelle, enı nosso projeto, em virtude de seus recursos e facilidade de uso e também pela disponibilidade de ferramentas de desenvolvimento e verificação.

Na primeira especificação, usamos o modelo de Rede Predicado-Ação, pois sua representação gráfica é didática e as ferramentas de desenvolvimento permitem análise completa da parte de controle do protocolo. Nesta fase, no entanto, não foi explicitada a manipulação dos dados, que a linguagem Estelle trata de maneira mais fácil e apropriada. O modelo também não foi verificado com ferramentas específicas, servindo apenas como guia para a especificação em Estelle, que foi testada e verificada. Resumindo: a especificação com Rede Predicado-Ação foi importante porque permitiu uma verificação inicial e auxiliou na especificação em Estelle. Mostramos, na figura 6, a Rede oAção da primitiva ManagerInit. As demais primitivas são mostradas em [14].

Uma vez especificado o controle do protocolo com o modelo de Rede Predicado-Ação, passamos para a manipulação de dados com a linguagem de descrição formal Estelle [15]. Esta linguagem é mais apropriada para a representação do protocolo porque apresenta uma estrutura de máquina de estado, o que permitiu aproveitar o modelo em Rede Predicado-Ação inicial.

A etapa seguinte foi a simulação para verificar o correto funcionamento do protocolo.

\subsection{ESPECIFICAÇÃO FORMAL}

O modelo implementado em linguagem Estelle é mostrado na figura 7. O módulo SGH pode ser instanciado quantas vezes forem necessárias, neste caso, sete vezes. O módulo Usuário permite a interface com o operador, possibilitando a montagem das SDUs (Service Data Units), conforme a especificação do serviço. O módulo CMIS enula o funcionamento de uma camada que oferece o serviço de gerenciamento, utilizando o protocolo CMIP. Este módulo apenas estabelece a comunicação entre os diversos módulos SGH, copiando as mensagens do módulo remetente para o módulo destinatário. 


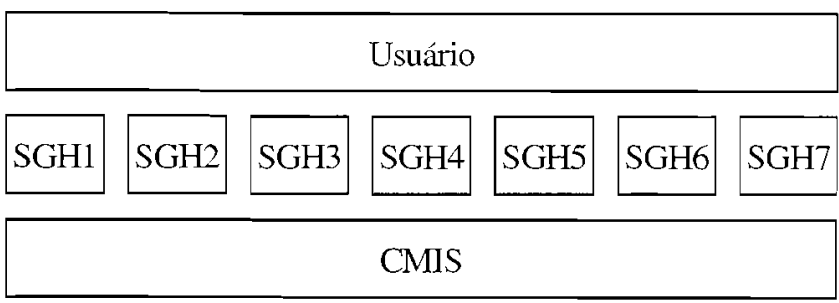

Figura 7. Modelo de simulação em Estelle

\subsection{SIMULAÇÃO E TESTES}

Os testes de simulação realizados tentaram atingir todas as situações previstas em nosso modelo de protótipo. O primeiro teste foi a iniciação do sistema hierárquico, esquematicamente mostrado na figura 8 .

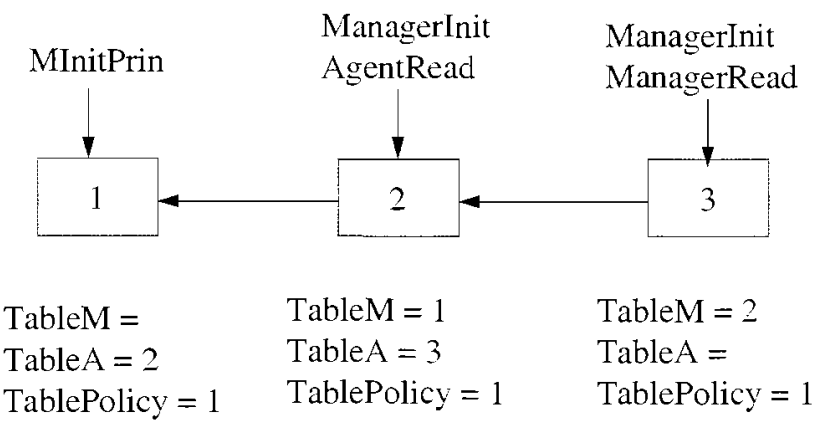

Figura 8. Teste de inicialização do sistema

Imiciamos o gerente $1 \mathrm{com}$ a primitiva MInitPrin. depois iniciamos o gerente $2 \mathrm{em}$ relação ao gerente 1 , com a primitiva ManagerInit, e o gerente 3 em relação ao gerente 2 , novamente com a primitiva ManagerInit. Após essa iniciação, pudemos testar o funcionamento através das primitivas ManagerRead, no gerente 3, e AgentRead, no gerente 2. Como resultado deste teste, a tabela TableM do gerente 3, lida através da primitiva ManagerRead, apresentou os gerentes 2 e 1 , coerente com a seqüência de início do sistema. A leitura da tabela TableA do gerente 2, lida através da primitiva AgentRead, apresentou apenas o gerente 3 , coerente com os resultados esperados.

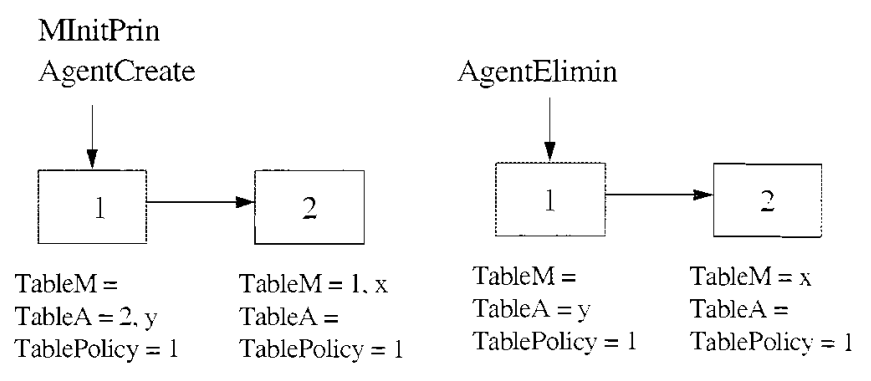

Figura 9. Teste de criação e exclusão de Agentes

O segundo teste foi a criação e exclusão de um elemento, utilizando as primitivas AgentCreate e AgentElimin, que é esquematicamente mostrado na figura 9. Com a primitiva MInitPrin, iniciamos o gerente 1 como gerente principal. Após isso, criamos o elemento 2 através da primitiva AgentCreate. Verificamos o funcionamento desta primitiva através das primitivas ManagerRead no elemento 2 e AgentRead no elemento 1. Nesta configuração, executamos a eliminação deste elemento através da primitiva AgentElimin e, novamente, verificamos o funcionamento através das primitivas ManagerRead no elemento 2 e AgentRead no elemento 1.

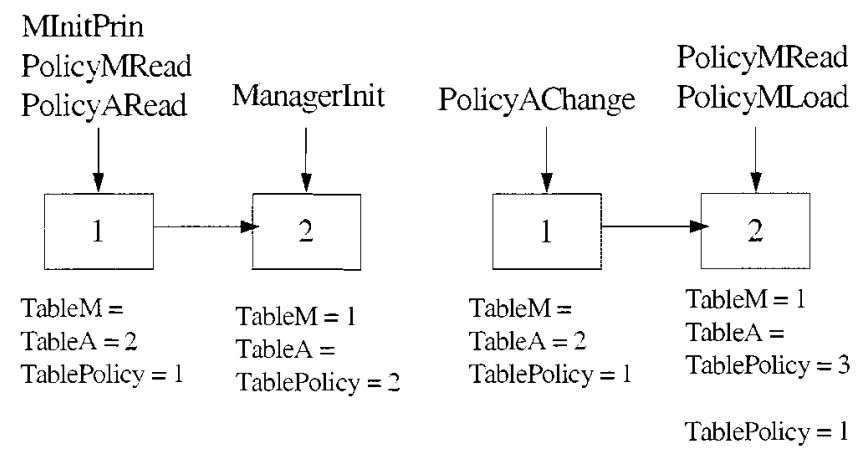

Figura 10. Teste de gerência de diretrizes

O terceiro teste ocupou-se da gerência de diretrizes. testando todas as suas primitivas. Este teste é mostrado na figura 10. Iniciamos SGHl como gerente principal e valor de diretriz 1, utilizando-se a primitiva MInitPrin. Iniciamos SGH2 como agente de SGH1 e com valor de diretriz 2, utilizando-se a primitiva ManagerInit. Realizamos uma leitura de diretrizes locais em SGH1, com a primitiva PolicyMRead. Fizemos a leitura das diretrizes do agente SGH2 através de SGH1, com a primitiva PolicyARead. Alteramos a diretriz do agente $\mathrm{SGH} 2$ para valor de diretriz 3 através de SGH1, com a primitiva PolicyAChange. O funcionamento desta pôde ser testado com a primitiva PolicyMRead em SGH2. Finalmente, fizemos uma atualização das diretrizes de $\mathrm{SGH} 2$ em relação às diretrizes de SGH1, através da primitiva PolicyMLoad, que tornou as diretrizes de SGH1 e SGH2 iguais ao valor 1.

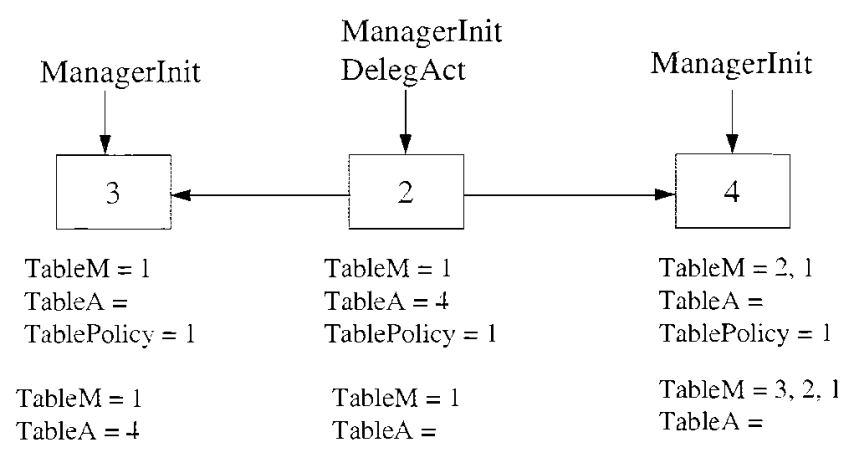

Figura 11. Teste de delegação

O quarto teste simulou uma delegação, que é mostrada na figura 11. Iniciamos o gerente SGH1 com dois agentes $\mathrm{SGH}_{2}$ e $\mathrm{SGH}_{3}$, através das primitivas MInitPrin e ManagerInit. Criamos o agente SGH4 relacionado ao gerente SGH2, novamente através da primitiva ManagerInit. Realizamos uma delegação do agente SGH4 do gerente SGH2 para o gerente SGH3, utilizando-se a primitiva DelegAct. Seu funcionamento foi verificado pela leitura da tabela de agentes de SGH2 e SGH3, utilizando-se a primitiva AgentRead, que mostrou a transferência do agente $\mathrm{SGH} 4$. 
A última etapa de testes foi a verificação exaustiva de todas as sequiências de testes produzidas pela ferramenta Gerador de Sequiência de Testes. Esta ferramenta gera todas as combinações de estado-transição do modelo de serviço, que é verificada através do Simulador. Com este teste, pudemos fazer uma validação inicial do protocolo proposto, pois foi garantida a inexistência de deadlocks durante seu funcionamento.

\subsection{IMPLEMENTAÇÃO SEMI-AUTOMÁTICA}

Após a simulação, procedeu-se à implementação semiautomática do $\mathrm{SGH}$, trabalho desenvolvido por DELFINO [16]. Para tal, foi utilizado, dentro do ambiente de projeto de protocolos em Estelle, o programa denominado IPDE (Implementador de Protocolos Descritos em Estelle). Este transforma uma especificação em linguagem de descrição formal diretamente em código executável.

Como a implementação é, a princípio, transparente ao usuário, foram necessárias algumas modificações na especificação, de modo a tornar a execução do programa mais amigável. Assim, criou-se o recurso de disparo de transiçôes pelo teclado e uma rotina de conversão do status de resposta das SDUs, que antes eram exibidas como mensagens numéricas, para uma mensagem textual na tela de erro, especificando o tipo de erro ou execução bem sucedida.

Uma vez que a forma de compilação Estelle é a mesma, tanto para o simulador como para o implementador, os testes realizados foram semelhantes aos feitos no simulador, mostrados na seção 4.4. Verificamos que todos os testes realizados tiveram resultados satisfatórios, exibindo mensagens de execução bem sucedida, alterando de forma adequada as tabelas de gerentes, agentes e diretrizes envolvidas no processo. Isto foi verificado tanto para iniciação de gerentes, criação e eliminação de agentes, testes com diretrizes e delegação.

\section{CONCLUSÃO}

Este trabalho apresentou uma proposta para um sistema hierárquico adequado ao gerenciamento de grandes redes de telecomunicações. Analisamos várias propostas encontradas na literatura e utilizamos os conceitos para um projeto de sistema hierárquico coerente e flexível. Esta flexibilidade, que advém em grande parte da forma independente com que - SGH opera nos diversos elementos da rede, não provoca, no entanto, perda de controle do sistema. O uso criterioso de diretrizes garante ao conjunto coerência e ausência de conflitos. Além disso, a possibilidade de delegação de gerenciamento confere ao sistema grande estabilidade e tolerância a falhas.

O protocolo SGH proposto não apenas pode controlar qualquer quantidade de dispositivos, como também pode adequar-se dinamicamente a qualquer alteração na quantidade e localização de objetos gerenciados. A facilidade de modificar-se e a arquitetura adaptável capacitam-no para o gerenciamento de dispositivos de comunicação móvel. Este trabalho seguiu as idéias básicas definidas pelo ITU-T e produziu uma aplicação consistente com o modelo TMN.
O sistema SGH oferece também recursos de segurança. Como cada gerente enxerga apenas a camada inferior e os critérios para troca de gerente são rigidamente definidos, então a capacidade de acesso de cada gerente pode ser limitada em qualquer nível. Além disso, o uso de técnicas de criptografia e autenticação, disponíveis no protocolo CMIP, garante segurança de acesso ao gerenciamento da rede.

O uso do modelo em Rede Predicado-Ação e da linguagem de especificação formal Estelle facilitou o trabalho de codificação. A especificação formal foi a maneira adequada para descrever o sistema em alto nível de abstração, podendo futuramente ser convertido para código de linguagem de programação, através de ferramentas apropriadas.

A característica hierárquica desta proposta possibilita que o conceito seja aplicado a uma rede de grande porte com cada gerente controlando um número limitado de nós. $O$ modelo de gerenciamento hierárquico permite recursos de escalabilidade para uma rede con grande número de elementos [6]

Como trabalho futuro, nesta linha. acreditamos que seria relevante e necessário o aprofundamento da gerência de diretrizes. Esta técnica possibilita a diminuição da quantidade de mensagens trocadas entre gerentes e, consequientemente, a diminuição de tempo na execução de comandos de configuração. A utilização de Inteligência Artificial e Lógica Fuzzy poderia permitir um tratamento de diretrizes eficiente e rápido. Assim, seria possível que cada gerente do sistema pudesse tomar grande parte das decisões autonomamente, diminuindo inclusive a necessidade de interferência humana.

\section{REFERÊNCIAS}

[1] ITU-T - International Telecommunication Union Telecommunication Standardzation SeCtor. Recommendation M.3010: Principles for a Telecommunications Management Network. ITU-T, Oct. 1992.

[2] CASE. J. D., FEDOR, M. S., SCHOFFSTALL, M. L., at al. A simple network management protocol (SNMP). RFC 1157. USA, May 1990.

[3] CASE, J., McCLOGHRIE, K., ROSE. M., et al. Manager-toManager Management Information Base. RFC 1451. USA. Apr. 1993.

[4] CASE, J. D., McClOGHRIE. K., ROSE, M. T., at al. Introduction to version 2 of the Intemet-standard Network Management Framework. RFC 1441. USA, Apr. 1993.

[5] BLACK, U. D. Network Management Standards - SNMP, CMIP, TMN, MIBs and Object Libraries - $2^{\text {nd }}$ Edition. 2 ed. McGraw-Hill. USA, 1995.

[6] SIEGL, M. R.. TRAUSMUTH, G. Hierarchical Network Management - A Concept and its Prototype in SNMP12. Proceedings of 6 th Join European Network Conference. Wien, May 1995.

[7] SCHALLER, N. H. "A concept for hierarchical, decentralized management of the physical configuration in the Internet", In: Proceedings of KiVS 95. Chemnitz, 20-24 Sep. 1995.

[8] SLOMAN, M., MAGEE, K., TWIDLE. $K$., at al. "An arquitecture for managing distributed systems", In: Proceedings of $4^{\text {th }}$ IEEE Workshop on Future Trends of Distributed Computing Systems. Lisboa. pp. 40-46, 22-24 Sep. 1993. 
[9] WIES, R. "Policy Definition and Classification: Aspects, Criteria and Examples". In: Proceedings of the IFIP/IEEE Intemational Workshop on Distributed Systems: Operation and Management. Toulouse-France, 10-12 Oct. 1994.

[10] GOLDSZMIDT, G., YEMINI, Y.. "Distributed management by delegation", In: Proceedings of $15^{\text {th }}$ International Conference on Distributed Computing Systems. New York, Jun. 1995.

[11] MOFFETT. J. D., SLOMAN, M. S. "Delegation of authority". In: IFIP 2nd Intemational Symposium on Integrated Network Management. Washington, pp. 595-606, Apr. 1991.

[12] ISO - INTERNATIONAL ORGANZATION FOR STANDARDIZATION. 9596 Information Technology, Open System Interconnection - Common Management Information Protocol - Part 1: Specification. ISO/IEC. May 1990.

[13] ITU-T - INTERNATIONAL TELECOMMUNCATION UNION TEle-COMmunication STANDARDization SEctor. Recommen-dation M.3100: Generic Network Information Model. ITU-T, Oct. 1992.

[14] FERNANDEZ. M. P. Protocolo para Gerenciamento Hierárquico de Redes de Telecomunicações (TMN). Tese M.Sc., COPPE/UFRJ, Rio de Janeiro, RJ, Brasil, janeiro 1998.

[15] DIAZ, M., at al. The Formal Description Technique ESTELLE. 1 ed. North-Holland. USA, 1989.

[16] DELFINO, G. M. Verificação e Implementação do Protocolo de Gerenciamento Hierárquico (SGH). Projeto Final de Curso. Departamento de Eletrônica EE/UFRJ, agosto 1999.

[17] ISO - INTERNATIONAL ORGANIZATION FOR STANDARDIZATION. 9596 Open System Interconnection - Common Management Information Protocol - Part 1: Specification. ISO/IEC, May 1990.
Marcial Porto Fernandez é graduado em Engenharia Eletrônica pela Universidade Federal do Rio de Janeiro (UFRJ), em 1988. Recebeu M.Sc. em Engenharia Elétrica no Grupo de Teleinformática e Automação (GTA) na COPPE/UFRJ em 1998; atualmente é estudante D.Sc. no GTA/COPPE/UFRJ. Suas áreas de interesse são Gerenciamento de Redes, Gerenciamento Baseado em Políticas e Diffserv.

Gardel Moreira Delfino é graduado em Engenharia Eletronica pela Universidade Federal do Rio de Janeiro (UFRJ), em 1998. O projeto de final de curso foi o módulo de usuário do Sistema de Gerenciamento Hierárquico. Atualmente é estudante M.Sc. no Grupo de Teleinformática e Automação (GTA) na COPPE/UFRJ. Suas áreas de interesse são linguagens de especificação formal de protocolos, particularmente Estelle.

Aloysio de Castro P. Pedroza recebeu o doutorado em Engenharia Elétrica e Ciência da Computação em 1985 em l'Universite Paul-Sabatier/LAAS, Toulouse. França; o M.Sc. em Engenharia Elétrica em 1980 na COPPE/UFRJ, Rio de Janeiro, e Engenharia Eletrônica em 1975, na UFRJ, Rio de Janeiro. Atualmente ele é professor adjunto da UFRJ, Rio de Janeiro. Suas áreas de pesquisa são especificação. verificação e implementação de software e hardware de sistemas distribuídos. 